\title{
Bose-Einstein condensation on a permanent-magnet atom chip
}

\author{
C. D. J. Sinclair, E. A. Curtis, I. Llorente Garcia, J. A. Retter, ${ }^{*}$ B. V. Hall, ${ }^{\dagger}$ S. Eriksson, B. E. Sauer, and E. A. Hinds ${ }^{\ddagger}$ \\ Centre for Cold Matter, Blackett Laboratory, Imperial College, Prince Consort Road, London SW7 2BW, United Kingdom
}

(Received 31 March 2005; published 21 September 2005)

\begin{abstract}
We have produced a Bose-Einstein condensate on a permanent-magnet atom chip based on periodically magnetized videotape. We observe the expansion and dynamics of the condensate in one of the microscopic waveguides close to the surface. The lifetime for atoms to remain trapped near this dielectric material is significantly longer than above a metal surface of the same thickness. These results illustrate the suitability of microscopic permanent-magnet structures for quantum-coherent preparation and manipulation of cold atoms.
\end{abstract}

DOI: 10.1103/PhysRevA.72.031603

PACS number(s): 03.75.Kk, 03.75.Be, 34.50.Dy, 39.25.+k

Atom chips are making rapid progress in the quantumcoherent manipulation of microscopic cold atom clouds [1-3] with a view to fundamental studies of quantum gases [4], interferometry [5], and quantum information processing [6]. Small-scale magnetic field patterns for atom chips can be made using either microfabricated current-carrying wires or microscopic structures of permanent magnetization. This second idea is attractive because there is no power dissipation in a permanent magnet and because very tight atom traps with oscillation frequencies of $\sim 1 \mathrm{MHz}$ (10-nm ground-state size) are possible [7]. Previous studies have used audiotape [8], floppy disks $[9,10]$, videotape [11], magnetic and magneto-optical films [7,12,13], and hard disks [14]. Until recently, permanently magnetized films were used only for millimeter- or centimeter-scale manipulation of atom clouds by reflection, but now cold atom clouds have been loaded into the microtraps $[12,15]$. For many applications of these magnetic microtraps, the next significant step is to prepare a Bose-Einstein condensate (BEC) on the chip as a source of coherent matter waves for interferometry or as a low-entropy reservoir for quantum information processing.

In this paper we describe the production of a BEC on a permanent-magnet atom chip made from videotape, which forms an array of waveguides. We have observed the propagation of the BEC along one of these guides. With the ends of the guide closed, we study center-of-mass and length oscillations of the trapped gas, demonstrating that the videotape chip is a practical way to manipulate cold atoms. We also show that the spin-relaxation time of the trapped atoms is significantly longer above the dielectric surface of the videotape than above a metal surface of the same thickness.

The waveguides of our atom chip are due to a sinusoidal pattern of magnetization written along the length of the videotape ( $\hat{x}$ direction) with the form $M_{0} \cos (k x) \hat{x}$, as illustrated in Fig. 1. This produces a field

\footnotetext{
*Present address: Laboratoire Charles Fabry de l'Institut d'Optique, UMR8501 du CNRS, 91403 Orsay Cedex, France.

${ }^{\dagger}$ Present address: Centre for Atom Optics and Ultrafast Spectroscopy, Swinburne University of Technology, Melbourne, Australia.

†Electronic address: ed.hinds@imperial.ac.uk
}

$$
\left(B_{x}, B_{y}\right)=B_{\text {sur }} e^{-k y}(-\cos (k x), \sin (k x)),
$$

where $B_{\text {sur }}$ is the field strength at the surface $(y=0)$. The waveguides appear, as shown in the background of Fig. 1, when a bias field $B_{\text {bias }}$ is added in the $x-y$ plane. Near the center of each guide, the magnetic field has a quadrupole structure with a gradient of field strength given by $B^{\prime}$ $=k B_{\text {bias }}$. An axial bias $B_{z}$ prevents the total field from going to zero at the center. For small-amplitude transverse oscillations this makes a harmonic trap with frequency

$$
2 \pi f_{r}=k B_{\text {bias }} \sqrt{\frac{\mu_{B} g_{F} m_{F}}{m B_{z}}},
$$

where $\mu_{B} g_{F} m_{F}$ is the usual factor in the Zeeman energy and $m$ is the mass of the atom.

Our atom chip is made using Ampex 398 Betacam SP videotape, which has a 3.5- $\mu \mathrm{m}$-thick magnetic layer containing iron-composite needles set in a glue and supported on a polymer ribbon $11 \mu \mathrm{m}$ thick. The magnetization has a period of $106 \pm 2 \mu \mathrm{m}$ and gives a surface field of $B_{\text {sur }}=11 \pm 1 \mathrm{mT}$ [15]. With a small bias field of $B_{\text {bias }}=0.1 \mathrm{mT}$ and $B_{z}$ $=0.1 \mathrm{mT}$, the atom guides lie $79 \mu \mathrm{m}$ from the surface of the chip. For ground state ${ }^{87} \mathrm{Rb}$ atoms in the $\left|F=2, m_{F}=2\right\rangle$ sublevel the transverse oscillation frequency is $760 \mathrm{~Hz}$. The bias field does not alter the magnetization of the videotape, which has very low susceptibility and high coercivity. Further details on permanent magnetic patterns may be found in Ref. [3].

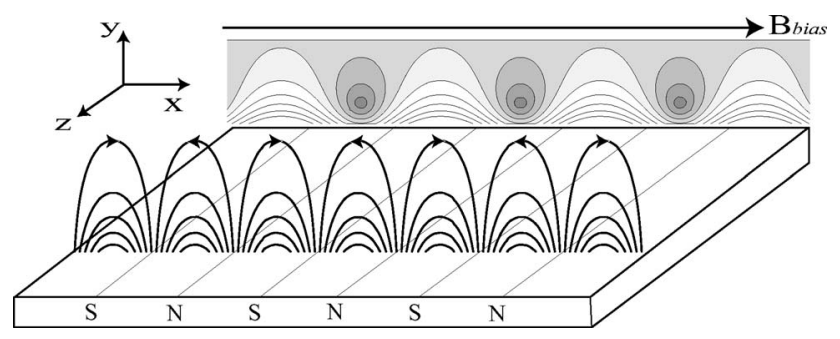

FIG. 1. Magnetic field lines produced by the magnetized videotape are shown in the foreground. A uniform bias field, $B_{\text {bias }}$, is added to this to make an array of atom guides. Contours of constant magnetic field strength are shown in the background. Circular contours enclose the lines of minimum field strength, where atoms are trapped. 


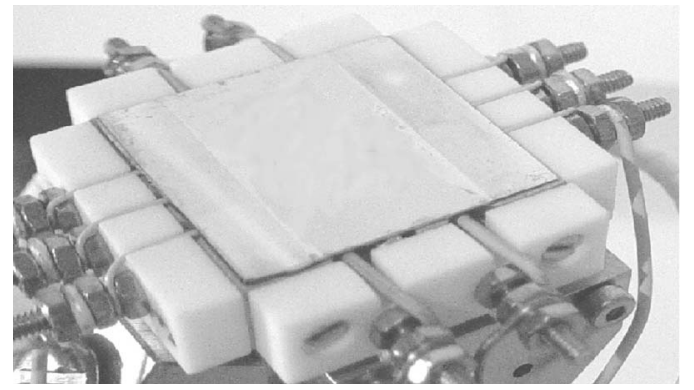

FIG. 2. Videotape atom chip assembly. The gold-coated surface is $2.5 \times 2.5 \mathrm{~cm}$. Wires underneath facilitate the loading and manipulation of atoms on the chip.

A piece of recorded videotape is glued to a glass coverslip and is coated with approximately $400 \mathrm{~nm}$ of gold to make the surface reflect $780 \mathrm{~nm}$ light. This allows the formation of a magneto-optical trap (MOT) by reflection [16] in order to collect and cool the atoms close to the videotape. The coverslip is then glued to a stainless steel base to form the assembly shown in Fig. 2. Underneath this, three wires run parallel to the $z$ direction. The central one, the "center wire," is used to transport cold atoms from the MOT into one of the videotape guides, while the outer two form a rf antenna for evaporative cooling. The two wires running along $x$ allow the guide to be pinched off to form a trap by making a field whose $z$ component rises to a maximum above each wire. The whole assembly is mounted in a vacuum chamber, where a remarkably low outgassing rate [17] allows the vacuum to reach $\sim 10^{-11}$ Torr. Further details of the construction are given in Ref. [15].

Cold ${ }^{87} \mathrm{Rb}$ atoms from a low-velocity intense source (LVIS) [18] are captured $4 \mathrm{~mm}$ from the surface by the reflection MOT. This is loaded for $20 \mathrm{~s}$ to collect typically $10^{9}$ atoms before the LVIS is turned off. The MOT is moved to $1.5 \mathrm{~mm}$ from the surface by ramping up an external bias field, while increasing the laser detuning from -15 to $-45 \mathrm{MHz}$, cooling the cloud to $50 \mu \mathrm{K}$. Next, the MOT light and quadrupole field are switched off, the atoms are optically pumped into the $\left|F=2, m_{F}=2\right\rangle$ state, and the cloud is recaptured in a purely magnetic trap, formed by passing $15 \mathrm{~A}$ through the center wire with a 1.4-mT bias field along $x$.

The bias field is ramped up to $4.4 \mathrm{mT}$ over $100 \mathrm{~ms}$, which increases the trap depth, compresses the cloud, and moves it to $160 \mu \mathrm{m}$ from the surface. An initial stage of forced evaporative cooling ensures the cloud is cold enough to load only one of the videotape microtraps. The rf field is swept over $6 \mathrm{~s}$ from 30 to $3.9 \mathrm{MHz}$, cooling the cloud to $\sim 15 \mu \mathrm{K}$. We continue to evaporate at $3.9 \mathrm{MHz}$ by reducing the centerwire current and the bias field over $4 \mathrm{~s}$. This gradually merges the wire trap with one of the videotape microtraps as the cloud approaches the surface. The wire current is then ramped to zero leaving the atoms confined entirely by the videotape microtrap at a temperature of order $10 \mu \mathrm{K}$.

A final rf sweep lasting 2 or $3 \mathrm{~s}$, typically down to $800 \mathrm{kHz}$, cools the cloud below its critical temperature. The transition to a condensate can be seen by releasing the cloud, allowing it to expand, and imaging its density by the absorption of light tuned to the $D 2(F=2 \rightarrow 3)$ transition. Figure 3(a)

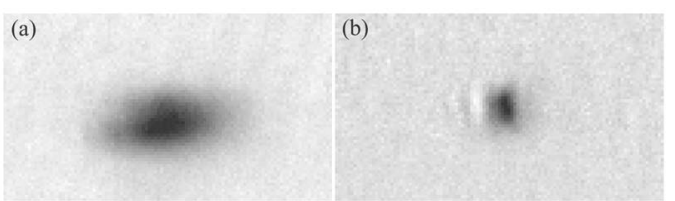

FIG. 3. Absorption images taken after $8.7 \mathrm{~ms}$ of free expansion. (a) Thermal cloud at $500 \mathrm{nK}$ with a final rf frequency of $820 \mathrm{kHz}$. (b) Condensate with a final rf frequency of $810 \mathrm{kHz}$. Image area is $0.9 \times 0.5 \mathrm{~mm}$.

shows a 500-nK thermal cloud imaged after $8.7 \mathrm{~ms}$ of expansion. The trap was $88 \mu \mathrm{m}$ from the surface with $f_{r}$ $=450 \mathrm{~Hz}$ and $f_{z}=15 \mathrm{~Hz}$ giving a prolate aspect ratio of $30: 1$. The isotropic thermal velocity distribution gives a nearly spherical image after expansion. By contrast, the condensate imaged in Fig. 3(b) is oblate, as expected for a condensate that is prolate in the microtrap. The cloud is released by switching off $B_{\text {bias }}$ in a time of 3 to $4 \mathrm{~ms}$.

An important feature of the atom chip is that it creates waveguides in which the atoms can propagate. We have used this as a better way to see the formation of the condensate. After evaporation, we open the ends of the trap by switching off the end wires. Now the cloud expands freely along the guide and we make absorption images of the atoms in situ. Figure 4(a) shows the Gaussian density profile of a 520-nK thermal cloud after $13.7 \mathrm{~ms}$ of expansion in a guide with $f_{r}$ $=580 \mathrm{~Hz}$. By contrast, Fig. 4(b) shows a similarly expanded BEC. Here the thermal component has a temperature of $330 \mathrm{nK}$ as obtained from a fit to the wings of the profile.

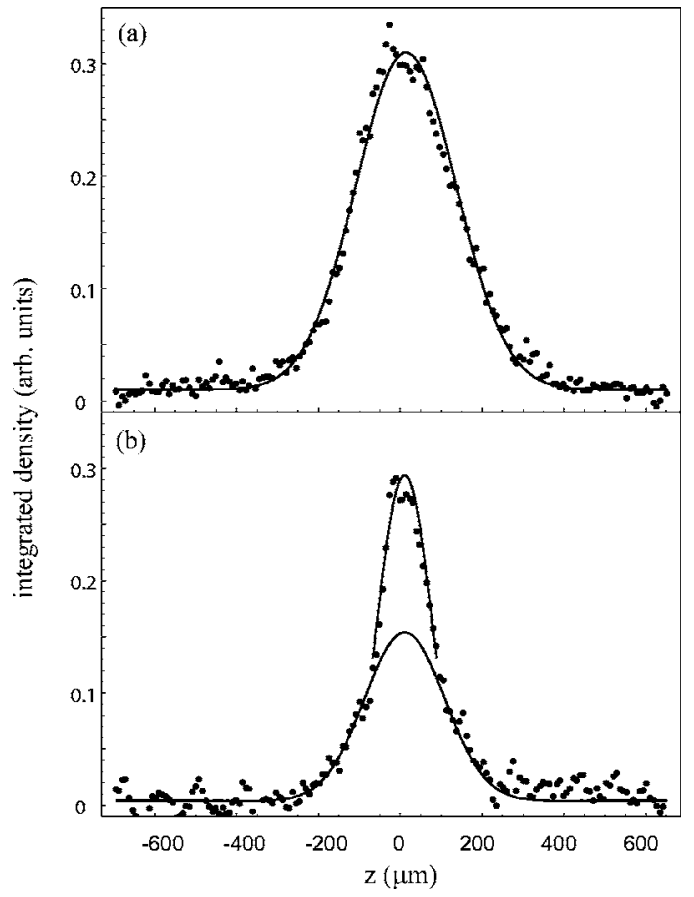

FIG. 4. Atom density profiles measured by absorption imaging after allowing the cloud to expand for $13.7 \mathrm{~ms}$ in a videotape waveguide. (a) Thermal cloud at $520 \mathrm{nK}$. (b) A partially condensed cloud with a thermal component at $330 \mathrm{nK}$. The parabolic central peak corresponding to the condensed fraction is $180 \mu \mathrm{m}$ wide. 


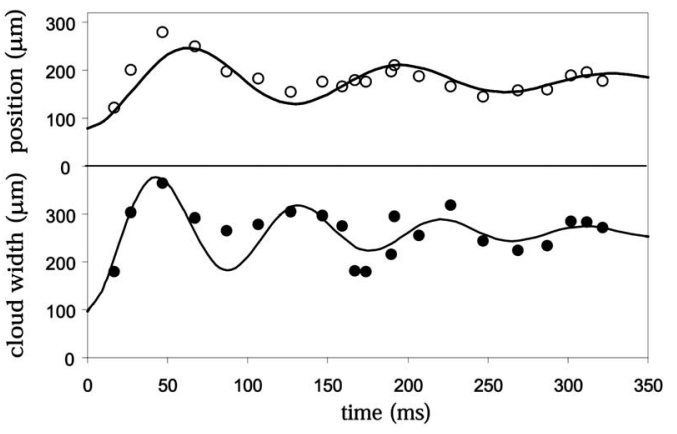

FIG. 5. Oscillations following a jump of the axial frequency. Open circles: center-of-mass oscillation. Filled circles: length oscillation. The solid curves are damped cosines. Each point represents a single destructive measurement.

Near the center we add a parabola of $180 \mu \mathrm{m}$ length to this in order to fit the region of the condensate. The expected condensate length can be found by the following rough estimate. Since a quarter of the cloud is condensed at $330 \mathrm{nK}$, we calculate that the initial trap $\left(f_{r}=320 \mathrm{~Hz}, f_{z}=15 \mathrm{~Hz}\right)$ has about $8 \times 10^{4}$ atoms in the condensate, with a Thomas-Fermi length of $90 \mu \mathrm{m}$. After $13.7 \mathrm{~ms}$ of expansion (see Eq. 11 of Ref. [19]), this length is expected to grow to $200 \mu \mathrm{m}$. Our measurement therefore indicates that the interaction with the thermal cloud may slightly impede the expansion, although we do not currently have enough accuracy in all the relevant parameters to be sure. In any case, the condensate length grows rapidly in the waveguide compared with a threedimensional expansion because there is no radial expansion to release the mean-field energy.

The permanent-magnet waveguides also offer a suitable setting in which to study more complex dynamics of the cloud. In order to demonstrate this possibility we jump the axial frequency to a lower value but do not remove the axial trapping entirely. This excites a length oscillation of the trapped cloud [20,21]. We also see an oscillation of its center of mass, due to a gravitational shift of the equilibrium position because the atom chip is not perfectly horizontal. Open circles in Fig. 5 show the center-of-mass motion, which is well described by a damped cosine of frequency $f_{z}$ $=7.6 \pm 0.4 \mathrm{~Hz}$ and $\sim 180 \mathrm{~ms}$ decay time. The damping is probably due to a slight roughness in the magnetic potential, which results from defects in the videotape material [15]. When the initial cloud is thermal, we measure a frequency for length oscillations of $2 f_{z}$, as expected. By contrast, if we prepare a partly condensed cloud before relaxing the trap we measure a slower breathing frequency. After a few tens of milliseconds, the atom density profiles no longer show a clear two-component distribution, indicating coupling between the condensed and thermal components. The filled circles in Fig. 5 show how the length of the cloud evolves over $300 \mathrm{~ms}$. The main features of the motion are once again described by a damped cosine with $180 \mathrm{~ms}$ decay time, but now the frequency is $11.2 \pm 0.4 \mathrm{~Hz}$. Within the accuracy of our measurements this frequency is consistent with $\sqrt{12 / 5} f_{z}=11.8 \pm 0.6 \mathrm{~Hz}$ as expected for a Bose gas in the hydrodynamic limit $[22,23]$. We anticipate that the damping

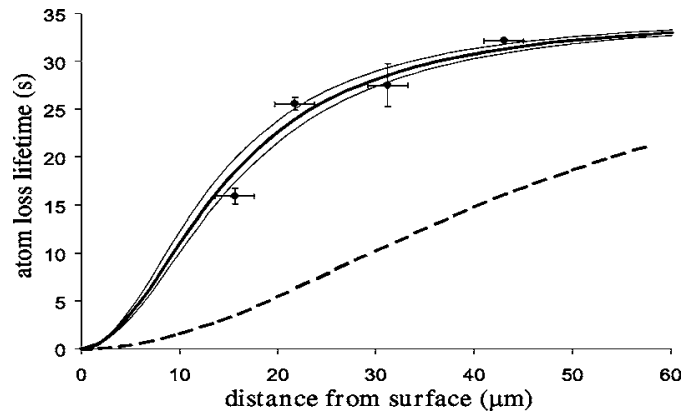

FIG. 6. Data: lifetimes for the loss of atoms trapped above the videotape atom chip with a 400-nm gold layer. Solid lines: the theory of Scheel et al. at temperature $350 \pm 50 \mathrm{~K}$. Dashed line: expected lifetime near a $4-\mu \mathrm{m}$-thick gold layer.

time of the length oscillation would be longer in a smoother guide and with a pure condensate. We are now studying the effects of roughness on the dynamics of cold clouds in these waveguides and we are pressing towards guides of higher radial frequency where aspects of one-dimensional manybody physics may be explored.

In contrast to other atom chips, the material of the videotape is an insulator, not a conductor. This is significant because the trapped atom loss due to spin flips is expected to be much less severe near an insulating surface [24] than it is near a metal $[25,26]$. Under conditions that apply to our experiment, we expect a lifetime $\propto \rho d^{2} / h$, where $\rho$ is the resistivity of the film, $h$ is its thickness, and $d$ is the atom-surface distance [1]. At the megahertz frequencies of relevance here, the resistivity of the videotape film is at least a million times larger than that of gold, so it is predicted that the spin flip rate above our chip should be due entirely to the 400-nm gold film on the surface. Scheel et al. have recently derived a rigorous formula for this rate $[27,28]$. Figure 6 shows the lifetime calculated according to Ref. [28] due only to the 400 -nm gold layer at the $350( \pm 50) \mathrm{K}$ temperature of the chip. Far from the chip the lifetime is $35 \mathrm{~s}$ due to collisions with the background gas. The data points show the longest thermal cloud lifetimes we have measured at various heights above the chip. They agree, confirming that the videotape itself makes no appreciable contribution to the loss rate. For comparison, the dashed line shows the calculated lifetime near a $4-\mu \mathrm{m}$-thick gold layer, which is the total thickness of our magnetic layer plus the gold coating, and is also typical of the gold thickness used to make atom chips with currentcarrying wires. This result shows that permanent magnets may be preferable to metal atom chips in applications where the atoms' quantum states decohere as a result of magnetic field fluctuations.

In this paper we have shown that a long thin BEC can be prepared in a microtrap formed by a videotape atom chip and can be manipulated in the waveguides of the chip. We have demonstrated a long spin-relaxation lifetime for atoms trapped in this way. These experiments show that permanentmagnet microstructures offer a powerful method for making atom microtraps with long lifetimes and tight confinement, 
which are relevant for applications involving lowdimensional quantum gases. To build more complicated quantum circuits, such as those required for quantum information processing, it will be necessary (and straightforward [7]) to write more elaborate magnetic patterns on the surface.
We acknowledge the technical assistance of Jon Dyne. This work is supported by the UK EPSRC Physics Program, the RCUK Basic Technology Program, the Royal Society, and the QGates, AtomChips, and FASTnet networks of the European Commission.
[1] R. Folman, P. Krüger, J. Schmiedmayer, J. Denschlag, and C. Henkel, Adv. At., Mol., Opt. Phys. 48, 263 (2002).

[2] J. Reichel, Appl. Phys. B: Lasers Opt. 74, 469 (2002).

[3] E. A. Hinds and I. G. Hughes, J. Phys. D 32, R119 (1999).

[4] Quantum Gases in Low Dimensions, edited by L. Pricoupenko, H. Perrin, and M. Olshanii, special issue of J. Phys. IV 116, 1 (2004).

[5] Y.-J. Wang, D. Z. Anderson, V. M. Bright, E. A. Cornell, Q. Diot, T. Kishimoto, M. Prentiss, R. A. Saravanan, S. R. Segal, and S. Wu, Phys. Rev. Lett. 94, 090405 (2005).

[6] T. Calarco, E. A. Hinds, D. Jaksch, J. Schmiedmayer, J. I. Cirac, and P. Zoller, Phys. Rev. A 61, 022304 (2000).

[7] S. Eriksson, F. Ramirez-Martinez, E. A. Curtis, B. E. Sauer, P. W. Nutter, E. W. Hill, and E. A. Hinds, Appl. Phys. B: Lasers Opt. 79, 811 (2004).

[8] T. M. Roach, H. Abele, M. G. Boshier, H. L. Grossman, K. P. Zetie, and E. A. Hinds, Phys. Rev. Lett. 75, 629 (1995).

[9] I. G. Hughes, P. A. Barton, T. M. Roach, M. G. Boshier, and E. A. Hinds, J. Phys. B 30, 647 (1997).

[10] C. V. Saba, P. A. Barton, M. G. Boshier, I. G. Hughes, P. Rosenbusch, B. E. Sauer, and E. A. Hinds, Phys. Rev. Lett. 82, 468 (1999).

[11] P. Rosenbusch, B. V. Hall, I. G. Hughes, C. V. Saba, and E. A. Hinds, Phys. Rev. A 61, 031404(R) (2000).

[12] I. Barb, R. Gerritsma, Y. T. Xing, J. B. Goedkoop, and R. J. C. Spreeuw, Eur. Phys. J. D 35, 75 (2005).

[13] D. C. Lau, R. J. McLean, A. I. Sidorov, D. S. Gough, J. Koperski, W. J. Rowlands, B. A. Sexton, G. I. Opat, and P. Hannaford, J. Opt. B: Quantum Semiclassical Opt. 1, 371 (1999).
[14] B. Lev, Y. Lassailly, C. Lee, A. Scherer, and H. Mabuchi, Appl. Phys. Lett. 83, 395 (2003).

[15] C. D. J. Sinclair, J. A. Retter, E. A. Curtis, B. V. Hall, I. Llorente Garcia, S. Eriksson, B. E. Sauer, and E. A. Hinds, Eur. Phys. J. D 35, 105 (2005).

[16] J. Reichel, W. Hänsel, and T. W. Hänsch, Phys. Rev. Lett. 83, 3398 (1999).

[17] S. A. Hopkins, E. A. Hinds, and M. G. Boshier, Appl. Phys. B: Lasers Opt. 73, 51 (2001).

[18] Z. T. Lu, K. L. Corwin, M. J. Renn, M. H. Anderson, E. A. Cornell, and C. E. Wieman, Phys. Rev. Lett. 77, 3331 (1996).

[19] Y. Castin and R. Dum, Phys. Rev. Lett. 77, 5315 (1996).

[20] D. S. Jin, J. R. Ensher, M. R. Matthews, C. E. Wieman, and E. A. Cornell, Phys. Rev. Lett. 77, 420 (1996).

[21] H. Ott, J. Fortágh, S. Kraft, A. Günther, D. Komma, and C. Zimmermann, Phys. Rev. Lett. 91, 040402 (2003).

[22] A. Griffin, W.-C. Wu, and S. Stringari, Phys. Rev. Lett. 78, 1838 (1997).

[23] D. M. Stamper-Kurn, H.-J. Miesner, S. Inouye, M. R. Andrews, and W. Ketterle, Phys. Rev. Lett. 81, 500 (1998).

[24] D. M. Harber, J. M. McGuirk, J. M. Obrecht, and E. A. Cornell, J. Low Temp. Phys. 133, 229 (2003).

[25] M. P. A. Jones, C. J. Vale, D. Sahagun, B. V. Hall, and E. A. Hinds, Phys. Rev. Lett. 91, 080401 (2003).

[26] Y. J. Lin, I. Teper, C. Chin, and V. Vuletić, Phys. Rev. Lett. 92, 050404 (2004).

[27] P. K. Rekdal, S. Scheel, P. L. Knight, and E. A. Hinds, Phys. Rev. A 70, 013811 (2004).

[28] S. Scheel, P. K. Rekdal, P. L. Knight, and E. A. Hinds, e-print arXiv: quant-ph/0501149. 